\title{
Haemodialysis in Poisoning by Sea-snake Venom
}

\author{
VISITH SITPRIJA, RAMPAI SRIBHIBHADH, CHANYO BENYAJATI
}

\section{Summary}

Myoglobinuria, renal failure, and muscular weakness or paralysis were noted in two patients following sea-snake bite. A pathological study by biopsy showed renal tubular necrosis and myonecrosis. Both patients were treated by haemodialysis, and there was a remarkable improvement in the muscular symptoms. Recovery was complete in both cases. It is believed that muscular paralysis was the effect of hyperkalaemia and uraemia on the muscles sensitized by the toxic effect of the sea-snake venom. Removal of potassium and uraemic factors by dialysis thus led to the rapid improvement of the muscular activity. Haemodialysis seems to be a useful alternative method to sea-snake antivenom in the treatment of sea-snake bite poisoning.

\section{Introduction}

Renal failure after sea-snake bite is usually associated with myoglobinuria (Marsden and Reid, 1961). Muscular symptoms, including pains and paralysis or weakness, constitute the main clinical features apart from renal failure (Reid, 1961). Pathological study has shown myonecrosis, and this was thought to be responsible for the development of muscular symptoms. We report two cases in which acute renal failure with muscular weakness and paralysis developed after sea-snake bite. Haemodialysis, performed for acute renal failure, remarkably improved the muscular symptoms.

\section{Case 1}

A Thai fisherman aged 19 was bitten on the left leg by a sea-snake four days before admission to Chulalongkorn Hospital in Bangkok. Half an hour after the bite he had stiffness of the right hand which gradually progressed to involve the left and then the whole of the upper and lower extremities. Within two hours, he became completely paralysed and was unable to open his mouth. The muscles of the extremities were painful to touch. He was admitted to a local hospital, where conservative treatment was given without any improvement. On the first day it was noted that urine became scanty and was dark brown. The urine volume remained low on the subsequent days. He was therefore transferred to Chulalongkorn Hospital for management of presumed renal failure.

On admission he was unable to move, but appeared to be in no distress. The blood pressure was $130 / 88 \mathrm{~mm} \mathrm{Hg}$, body temperature $36.6^{\circ} \mathrm{C}$, respiration $18 / \mathrm{min}$, and pulse rate $64 / \mathrm{min}$. Ptosis was present. Muscular paresis was more pronounced in the proximal groups than in the distal. Deep tendon reflexes were absent. Appreciation of sensation was, however, normal. He could barely open his mouth. An electromyogram of the left deltoid muscle showed polyphasic motor unit potentials of short duration and low voltage suggestive of myopathy (Fig. 1). The urinary output on the first day of admission was $420 \mathrm{ml}$. The urine was dark brown, acid in reaction, with a specific gravity of 1010. A few erythrocytes and red

Department of Medicine, Chulalongkorn Hospital Medical School, Bangkok, Thailand

VISITH SITPRIJA, M.D., PH.D., Assistant Professor of Medicine RAMPAI SRIBHIBHADH, M.D., Instructor of Medicine CHANYO BENYAJATI, M.D., Assistant Professor of Medicine blood cells casts were observed. Myoglobin in the urine was detected by spectrophotometric technique. The haemoglobin was $11 \mathrm{~g} / 100 \mathrm{ml}$ and a white blood count was $12,000 / \mathrm{mm}^{3}(77 \%$ neutrophils and $23 \%$ lymphocytes). The blood urea nitrogen was $80 \mathrm{mg} / 100 \mathrm{ml}$, serum creatinine $5 \mathrm{mg} / 100 \mathrm{ml}$, serum sodium 126 $\mathrm{mEq} / \mathrm{l}$, potassium $8.0 \mathrm{mEq} / 1$, chloride $91 \mathrm{mEq} / 1, \mathrm{CO}_{2}$ content $22 \mathrm{mEq} / 1$., and plasma osmolarity $290 \mathrm{mOsm} / \mathrm{kg}$. An electrocardiogram showed peaked $T$ waves. Because of hyperkalaemia haemodialysis was performed for a period of four hours. At the end of dialysis the serum potassium was $4 \mathrm{mEq} / \mathrm{l}$. and the blood urea nitrogen $40 \mathrm{mg} / 100 \mathrm{ml}$. There was a pronounced clinical improvement during and immediately after dialysis.

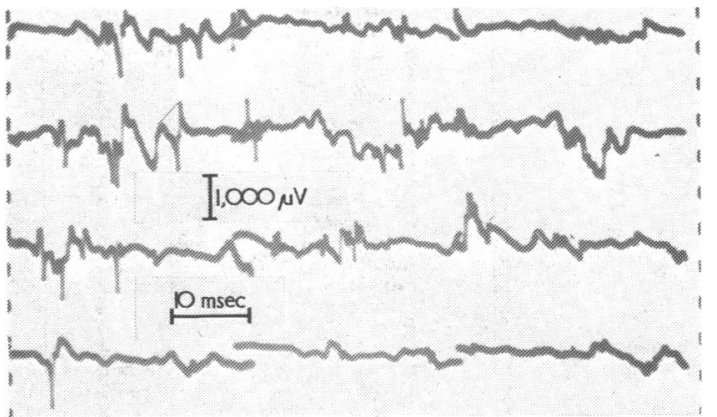

FIG. 1-Case 1. Electromyogram of left deltoid muscle before dialysis, showing low voltage, short duration, and polyphasic units (1:1,000 microvolts).

The patient was able to lift his arms and legs two hours after dialysis was started. Ptosis disappeared at the end of dialysis. On the next day he was able to walk. The electromyogram showed increased voltage, still with an underlying myopathic pattern (Fig. 2). Muscular pains persisted for six days. The urinary output gradually rose. The diuretic phase was reached on the third hospital day when the urinary output was $1,000 \mathrm{ml} . / 24$ hours. Blood chemistry returned to normal on the 22nd day. The clinical course after the sixth hospital day was uneventful, and the patient was discharged 38 days after admission.

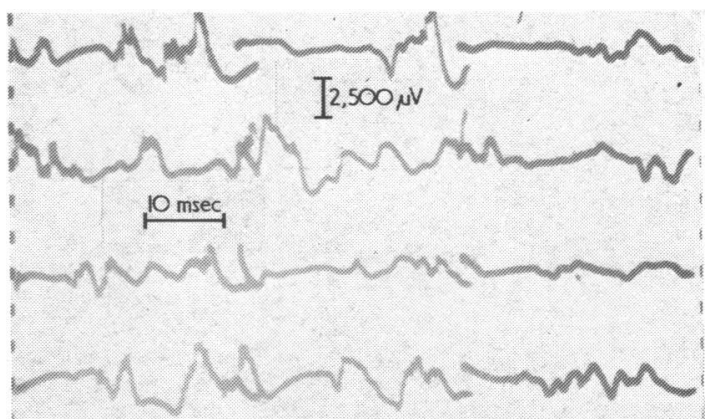

FIG. 2-Case 1. Electromyogram of same muscle as in Fig. 1 taken one day after dialysis showed increased voltage on a different scale (1:2,500 microvolts).

Percutaneous renal biopsy performed on the 20th hospital day showed tubular necrosis. A muscle biopsy specimen on the left gastrocnemius muscle obtained on the fourth hospital day showed degeneration of muscle fibres with loss of striations. 
Case 2

A man aged 22 was bitten on the right hand by a sea-snake three days before admission. Muscular symptoms, which included pains and weakness on the upper and lower extremities, developed half an hour after the bite. Only a small amount of dark brown urine was passed. He was treated in a rural hospital without any improvement and was transferred to Chulalongkorn Hospital.

On examination he showed no distress. Blood pressure was $128 / 80 \mathrm{~mm} \mathrm{Hg}$, respiration $20 / \mathrm{min}$, and pulse $72 / \mathrm{min}$. The body temperature was $37 \cdot 1^{\circ} \mathrm{C}$. Muscles were tender and there was weakness of both upper and lower extremities. Tendon reflexes were absent. Sensory function was normal. Laboratory data included a haemoglobin of $10.8 \mathrm{~g} / 100 \mathrm{ml}$, white blood count of $12,500 / \mathrm{mm}^{3}$ ( $80 \%$ neutrophils and $20 \%$ lymphocytes), a blood urea nitrogen of $100 \mathrm{mg} / 100 \mathrm{ml}$, serum creatinine $5 \cdot 1 \mathrm{mg} / 100 \mathrm{ml}$, serum sodium $136 \mathrm{mEq} / 1$., potassium $7.2 \mathrm{mEq} / 1$., CO., content $20 \mathrm{mEq} / 1$, and plasma osmolarity $315 \mathrm{mOsm} / \mathrm{kg}$. The urine was dark in colour and was positive for myoglobin by spectro-photometric examination. The specific gravity was 1011 . The sediment showed 5-6 red blood cells per high-power field. The 24-hour urine volume on the first hospital day was $210 \mathrm{ml}$. An electrocardiogram showed tall $T$ waves with a narrow base. Because of the rising serum potassium, blood urea nitrogen, dialysis, and creatinine haemodialysis was performed on the third day. The patient regained muscular strength during dialysis. At the end of dialysis, when the serum potassium was $2.8 \mathrm{mEq} / \mathrm{l}$., he could move his arms and legs with ease, and on the following day he was able to walk. There was a gradual return of tendon reflexes. Muscular pains disappeared on the sixth hospital day. He remained oliguric, necessitating a second haemodialysis on the seventh day. Diuresis began on the eighth day and later became more profuse with a gradual decline in blood urea nitrogen and creatinine. The remaining course was uneventful. The serum creatinine returned to normal on the 21 st hospital day.

Muscle biopsy obtained on the fourth hospital day showed myonecrosis. Renal biopsy performed on the 18th hospital day during the diuretic phases showed tubular necrosis.

\section{Discussion}

It is generally held that sea-snake venom in man is myotoxic, causing necrosis of striated muscles which results in muscular paralysis and myoglobinuria. Renal failure is common, and renal tubular necrosis has been observed at necropsy (Marsden and Reid, 1961). In our patients both tubular necrosis and myonecrosis were shown in the biopsy specimens. The electromyogram was consistent with myopathy. Both patients had myoglobinuria and oliguria on the first day of the bite. After transfer to Chulalongkorn Hospital acute renal failure was confirmed. Hyperkalaemia was a striking biochemical finding. Evidence of renal failure continued for 26 and 24 days respectively.

The muscular symptoms were similar to those previously described by Reid (1961). Muscular pain and weakness started 30 minutes after the bite and were more pronounced in proximal muscle groups. Ptosis and trimus were present in one patient. Tendon reflexes were absent. Pupillary light reflexes were intact and sensory function was normal.

Of clinical interest is the observation that haemodialysis dramatically improved the muscular symptoms. Both patients were able to walk the day following dialysis. Improvement of muscular activity was evident in the electromyogram in Case 1. Muscular pain, however, persisted for six days in both cases. This rapid improvement of muscular symptoms by dialysis indicates that myonecrosis was only partially responsible for the muscular symptoms. The clinical picture is best explained by the presence of a dialysable circulating substance (or substances) which inhibits muscular activity. Myonecrosis, usually incomplete, is responsible for the muscular pain which lasts longer (Marsden and Reid, 1961).

The nature of this circulating substance (or substances) cannot be identified from this report. Having a molecular weight of about 9,000 (Reid, 1961), sea-snake venom would not be dialysed, though its metabolites might be dialysable. Hyperkalaemia and uraemia further complicated the clinical picture. Both potassium and uraemic factors are readily dialysed. Hyperkalaemic paralysis has been noted in a patient with severe renal disease during spironolactone therapy for oedema (Herman and Rado, 1966). However, this is rarely observed. It is conceivable that a combination of all factors might contribute to the muscular symptoms. The myotoxic effect of the venom might sensitize the muscles to the paralytic effects of hyperkalaemia and uraemia. Removal of potassium and uraemic factors by dialysis thus resulted in rapid improvement of muscular activity.

It should be emphasized that death in sea-snake bite has been attributed to renal failure or to hyperkalaemia or to respiratory paralysis (Reid, 1961). The rapid improvement of muscular symptoms and the uraemic syndrome by haemodialysis in these patients is therefore of considerable clinical importance. The clinical course of poisoning can be shortened and death may be prevented by dialysis. The results of the treatment with dialysis are comparable to those observed in patients who received specific sea-snake antivenom (Reid, 1962). Haemodialysis thus appears to be a life-saving procedure for a sea-snake bite poisoning and constitutes an alternative treatment, especially in hospitals where sea-snake antivenom is not available. Whether or not the simpler procedure of peritoneal dialysis would be as effective as haemodialysis remains to be evaluated.

We are grateful to Dr. H. Alistair Reid for his interest and help and to Miss C. A. Bamberry for secretarial assistance.

\section{References}

Herman, E., and Rado, J. (1966). Archives of Neurology, 15, 74.

Marsden, A. T. H., and Reid, H. A. (1961). British Medical fournal, 1, 1290. Reid, H. A. (1961). British Medical Journal, 1, 1284.

Reid, H. A. (1962). British Medical fournal, 2, 576. 